\title{
Orientações às gestantes no pré-natal: a importância do cuidado compartilhado na atenção primária em saúde ${ }^{a}$
}

\author{
Guidelines to pregnant women: the importance of the shared care in primary health care \\ Directrices para las mujeres embarazadas: la importancia de la atención compartida en la atención \\ primaria de salud
}

Bruna Leticia Marques ${ }^{1}$ (1)

Yaná Tamara Tomasi ${ }^{2}$ D Suelen dos Santos Saraiva ${ }^{2}$ (1) Antonio Fernando Boing ${ }^{2}$ (D)

Daniela Savi Geremia ${ }^{1}$ (D)

1. Universidade Federal da Fronteira Sul.

Chapecó, SC, Brasil.

2. Universidade Federal de Santa Catarina, Programa de Pós-graduação em Saúde Coletiva. Florianópolis, SC, Brasil.
Autor correspondente: Bruna Leticia Marques. bmarquzz@gmail.com.

\section{Recebido em 30/03/2020.}

Aprovado em 17/06/2020.

DOI:https://doi.org/10.1590/2177-9465-EAN-2020-0098

\section{RESUMO}

Objetivo: Analisar a associação entre a adequação das orientações recebidas durante o pré-natal e o profissional que atendeu a gestante na maioria das consultas na Atenção Primária à Saúde. Método: Participaram 3.111 puérperas que realizaram pré-natal pelo Sistema Único de Saúde no Estado de Santa Catarina em 2019, através de questionário aplicado em ambiente hospitalar até 48 horas pós-parto. Analisou-se associação entre a variável de exposição principal e covariáveis, e o desfecho segundo profissional que atendeu no pré-natal. Resultados: As orientações mais frequentes foram os sinais de riscos na gestação $(80,3 \%)$ e riscos de automedicação $(76,9 \%)$. Observaram-se prevalências abaixo de $50 \%$ nas orientações sobre manejo adequado da amamentação $(45,9 \%)$ e possibilidade de visitar a maternidade antes do parto $(38,2 \%)$; ter recebido todas as orientações ao menos uma vez durante o pré-natal foi de 18,4\%. Gestantes atendidas na maioria das consultas pelos profissionais médico e enfermeiro apresentaram chance $41,0 \%$ maior de adequação às orientações, em comparação com aquelas atendidas exclusivamente por médicos. Conclusões e implicações para a prática: A prevalência de orientações dadas pelos profissionais de saúde às gestantes foi mais elevada quando o pré-natal foi mais compartilhado entre enfermeiros e médicos, em comparação ao atendimento majoritário por profissional de apenas uma profissão.

Palavras-chave: Cuidado Pré-Natal; Atenção Primária à Saúde; Promoção da

Saúde; Educação em Saúde; Equipe de Assistência ao Paciente.

\section{ABSTRACT}

Objective: To analyze the association between the adequacy of the guidelines received during prenatal care and the professional who assisted the pregnant woman in most consultations in Primary Health Care. Method: 3,111 puerperal women who underwent prenatal care by the Unified Health System in the State of Santa Catarina in 2019 participated, through a questionnaire applied in a hospital environment up to 48 hours postpartum. Association between the main exposure variable and covariates with the outcome according to the professional who carried out the prenatal care was analyzed. Results: The most frequent guidelines were the signs of risks during pregnancy (80.3\%) and risks of self-medication (76.9\%). Prevalences below $50 \%$ were observed in the guidelines on adequate breastfeeding management $(45.9 \%)$ and the possibility of visiting the maternity ward before delivery (38.2\%); having received all guidelines at least once during prenatal care was $18.4 \%$. Pregnant women assisted in most consultations by physicians and nurses had a $41 \%$ greater chance of adequacy in the guidelines compared to those assisted exclusively by physicians. Conclusions and implications for practice: The prevalence of guidelines given by health professionals for pregnant women was higher when prenatal care was more shared between nurses and physicians in comparison to the majority of care provided by professionals from only one profession.

Keywords: Prenatal Care; Primary Health Care; Health Promotion; Health Education; Patient Care Team.

\section{RESUMEN}

Objetivo: analizar la asociación entre la adecuación de las directrices recibidas durante la atención prenatal y el profesional que atendió a la mujer embarazada en la mayoría de las consultas en la Atención Primaria de Salud. Método: participaron 3.111 mujeres puérperas que recibieron la atención prenatal a través del Sistema Único de Salud en el Estado de Santa Catarina en 2019, a través de un cuestionario aplicado en un entorno hospitalario hasta 48 horas después del parto. Se analizó la asociación entre la variable de exposición principal y las covariables, y el resultado según el profesional que asistió en la atención prenatal. Resultados: las directrices más frecuentes fueron los signos de riesgos en el embarazo $(80,3 \%)$ y los riesgos de automedicación (76,9\%). Se observaron prevalencias inferiores al $50 \%$ en las directrices sobre el manejo adecuado de la lactancia materna (45,9\%) y la posibilidad de visitar la sala de maternidad antes del parto $(38,2 \%)$; han recibido todas las directrices al menos una vez durante la atención prenatal fue del $18.4 \%$. Las mujeres embarazadas atendidas en la mayoría de las consultas por profesionales médicos y enfermeras tuvieron un $41 \%$ más de posibilidades de adecuarse a las directrices en comparación con las que fueron atendidas exclusivamente por médicos. Conclusiones e implicaciones para la práctica: la prevalencia de las directrices otorgadas por los profesionales de la salud a las mujeres embarazadas fue mayor cuando la atención prenatal fue más compartida entre las enfermeras y los médicos en comparación con la mayoría de la atención prestada por profesionales de una sola profesión.

Palabras clave: Atención Prenatal; Atención primaria de salud; Promoción de la Salud; Educación en Salud; Grupo de Atención al Paciente. 


\section{INTRODUÇÃO}

Ações de saúde para a qualificação da atenção à mulher e à criança são prioritárias no desenho de políticas públicas, e têm promovido avanços na redução das mortalidades de mulheres e crianças. Entre as últimas décadas do século $X X$ e as primeiras décadas dos anos 2000, o Brasil conseguiu avanços expressivos na redução da mortalidade na infância, com o valor alcançando, em 2018, 14,4 óbitos de menores de cinco anos de idade a cada 1000 mil nascidos vivos. ${ }^{1}$ Os óbitos maternos também vêm apresentando redução, mas 60 óbitos a cada 100 mil nascidos vivos na razão de morte materna em 2015 foram insuficientes para o Brasil atingir a meta de reduzir o valor a 35 óbitos a cada 100 mil nascidos vivos, conforme previsto nos Objetivos de Desenvolvimento do Milênio. ${ }^{2}$ Assim, diferentes instâncias de governo e da sociedade civil têm se mobilizado para pensar e implementar ações por meio de políticas e programas com o objetivo de qualificar a atenção à criança e à mulher no país.

O acompanhamento pré-natal, por meio de ações preventivas, busca assegurar o saudável desenvolvimento da gestação e possibilitar o nascimento de um bebê saudável, com preservação de sua saúde e de sua mãe. Estudos têm demonstrado que um pré-natal qualificado está associado à redução de desfechos perinatais negativos, como baixo-peso e prematuridade, além de reduzir as chances de complicações obstétricas, como eclâmpsia, diabetes gestacional e mortes maternas. ${ }^{3,4}$ No entanto, apesar da alta cobertura de pré-natal entre as gestantes usuárias do Sistema Único de Saúde (SUS), estudo com amostra nacional realizado em 2011/2012 que analisou a adequação pré-natal conforme as recomendações do Ministério da Saúde, demonstrou que apenas $21,6 \%$ das mulheres receberam acompanhamento pré-natal considerado. ${ }^{5}$ Tal achado reforça que somente a alta cobertura de consultas no acompanhamento pré-natal não garante necessariamente a qualidade da assistência prestada.

Todas as orientações fornecidas pelos profissionais de saúde às gestantes durante o acompanhamento pré-natal são parte importante nesse processo de cuidado. ${ }^{6}$ Embora relevantes, no entanto, estudos nacionais têm identificado falha dos profissionais de saúde em oferecer orientações sobre a gestação, importância e técnicas para o aleitamento materno, como se preparar para o parto e cuidados básicos com o recém-nascido. Estudo que avaliou a qualidade da atenção pré-natal na rede básica de saúde do Brasil, ${ }^{7}$ identificou que apenas $60 \%$ das gestantes brasileiras atendidas no SUS receberam todas as orientações preconizadas durante o acompanhamento pré-natal.

Neste sentido, a Atenção Primária à Saúde (APS) configura-se como espaço estratégico para um pré-natal de baixo risco e de qualidade. No Brasil, a APS, norteada pela Política Nacional de Atenção Básica (PNAB), destaca que é competência da equipe de saúde o acolhimento e a atenção à saúde da gestante e da criança, englobando a prevenção de doenças, a promoção da saúde e o tratamento de agravos ocorridos durante o período gestacional até o período puerperal e os cuidados com a criança. Nesse cenário, a atuação compartilhada entre os profissionais da saúde possibilita diferentes olhares sobre as práticas no acompanhamento pré-natal, garantindo uma atenção integral e aumentando o potencial de resolutividade. .-10 $^{-1}$

No entanto, não há estudos no Brasil analisando se há diferenças na qualidade do pré-natal - em particular nas orientações às gestantes, segundo as recomendações do Ministério da Saúde - de acordo com a categoria profissional que realizou os atendimentos, e se a assistência por enfermeiros e médicos está associada com melhor orientação, quando comparada a que é prestada apenas por um profissional. Assim, o objetivo do presente estudo foi analisar a associação entre a adequabilidade das orientações recebidas durante o pré-natal e o profissional que atendeu a gestante na maioria das consultas.

\section{MÉTODO}

Trata-se de estudo quantitativo, do tipo transversal, realizado entre puérperas residentes no Estado de Santa Catarina. O estado possuía, em 2019, 295 municípios e população estimada de 7.164.788 mil habitantes. Sua rede assistencial conta com 1.947 Equipes de Estratégia de Saúde da Família (ESF), distribuídas em 748 Unidades Básicas de Saúde (UBS) em todo o estado; na rede assistencial hospitalar, conta com 1.109 leitos para parto SUS em 120 hospitais. , $^{5,6}$

A população deste estudo foi constituída por puérperas de qualquer idade que tiveram filhos nascidos em hospitais de Santa Catarina entre janeiro e agosto de 2019, e que atenderam aos seguintes critérios de inclusão: (1) ter residido em Santa Catarina durante toda a gestação; (2) ter realizado todas as consultas do pré-natal no SUS na rede básica de saúde ou em serviço especializado (pré-natal de alto risco); (3) ter realizado o parto em uma das 31 maternidades que em 2016 tiveram 500 ou mais partos via SUS; (4) ter tido filho nascido vivo, natimorto ou morto até 48 horas pós-parto, e que nasceram com mais de $500 \mathrm{~g} \mathrm{e}$ pelo menos 22 semanas de gestação.

Para composição da amostra, foram utilizados como parâmetros: nível de confiança de 95\%, margem de erro de 1,6\%, tamanho da população de 50 mil e, no intuito de majorar a amostra final, prevalência esperada do fenômeno de $50 \%$. Adicionou-se o valor de $5 \%$ ao total obtido para contemplar perdas e recusas. O número de entrevistas em cada maternidade foi definido de forma proporcional ao número de partos que a mesma realizou em 2016. A amostra total do estudo foi estimada em 3.665 puérperas a serem entrevistadas em 31 hospitais espalhados em 30 municípios do estado.

A coleta de dados ocorreu por meio da aplicação de questionário composto por 365 questões estruturadas em 11 blocos. Os dados foram coletados junto à puérpera, nos prontuários e também na caderneta de gestante. $O$ trabalho de campo foi desenvolvido por entrevistadores treinados pela equipe de pesquisa. A coleta de dados ocorreu em ambiente hospitalar, mediante entrevistas realizadas face a face até 48 horas após o nascimento do bebê, com o auxílio de tablets. Os dados eram armazenados na plataforma RedCap e enviados diariamente de cada município ao servidor central da pesquisa. Para o controle de qualidade dos dados, uma amostra aleatória de $10 \%$ das entrevistadas foi 
contatada novamente por meio de telefone, e a elas aplicou-se um questionário reduzido. ${ }^{11}$ Todas as variáveis do controle de qualidade mostraram concordância boa ou quase perfeita, sendo que seis das oito variáveis analisadas apresentaram Kappa de Cohen maior que 0,680 .

A variável de desfecho foi a adequação de orientações profissionais no pré-natal, categorizada em "adequado" ou "inadequado", sendo considerado adequado quando a puérpera informou ter recebido em pelo menos um momento, duranto seu pré-natal, as orientação descritas pelo Ministério da Saúde no Caderno de Atenção Básica n³2, de Atenção ao Pré-Natal de Baixo Risco, sobre: a importância da amamentação exclusiva até os seis meses; como fazer o manejo da amamentação; a importância de realização de atividades físicas durante a gestação; os riscos de automedicação e de se consumir álcool e tabaco durante a gestação; os sinais de risco que devem ser observados durante o período pré-natal; os sinais de início do trabalho de parto; existência de possibilidade de acompanhante durante o parto e o pós-parto e a possibilidade de visita à maternidade durante o pré-natal. ${ }^{12}$

Como variável de exposição principal, utilizou-se a pergunta: qual profissional atendeu a gestante na maioria das consultas de pré-natal (enfermeiro, médico ou enfermeiro/médico (ambos): Foram utilizadas as seguintes variáveis de ajuste: a) morar com marido ou companheiro (sim/não); b) idade em anos completos (16 a 20;21 a 24;25 a 34;35 ou mais); c) cor da pele autorreferida (branca; preta; parda), sendo que as categorias amarela e indígena foram excluídas, por representarem apenas $1 \%$ da amostra; d) escolaridade (ensino fundamental incompleto; fundamental completo; médio completo; superior completo); e) número de consultas de pré-natal (menor ou igual 6; 7 ou mais); e f) início do pré-natal (menor ou igual a 12 semanas de gestação; maior que 12)

Foram estimadas, primeiramente, as frequências absolutas e relativas das variáveis investigadas. Em seguida, por meio de regressão logística, foram calculados os odds ratio (OR) e testadas as associações entre as variáveis independentes e o desfecho em modelos brutos e ajustados. As variáveis foram incluídas de forma simultânea na análise ajustada, por meio do procedimento stepwise backward. A análise dos dados foi conduzida no software Stata versão 14.0 (StataCorp, Texas, USA).

A pesquisa possui aprovação do Comitê de Ética em Pesquisa com Seres Humanos (CEP) da Universidade Federal de Santa Catarina (UFSC), sob o parecer número 1.599.464, de20/06/2016, e seguiu os preceitos éticos, conforme preconizado pela Resolução n 466, de 12 de dezembro de 2012, do Conselho Nacional de Saúde.

\section{RESULTADOS}

Foram entrevistadas 3.580 puérperas, correspondendo a uma taxa de resposta de $96,7 \%$. Destas, 3.559 realizaram pré-natal; aquelas que possuíam completude de informação de todos os dados investigados somaram 3.111 e formarm a população deste estudo. A amostra foi constituída, em sua maior parte, por mulheres de 25 a 34 anos (46,2\%), que moravam com marido ou companheiro (81,3\%), que autorreferiram cor da pele branca $(63,7 \%)$ e com nível ensino médio ou superior completo (50,7\%) (Tabela 1).

Ao analisar o acompanhamento pré-natal, observou-se que $48,4 \%$ das gestantes foram atendidas no pré-natal majoritariamente somente por médico, 80,4\% realizaram sete ou mais consultas e $78,1 \%$ tiveram o início precoce do pré-natal até 12 semanas de gestação (Tabela 2).

Com relação às orientações recebidas durante o acompanhamento pré-natal, ao se analisar toda a amostra, observou-se que as mais comuns foram orientações sobre os sinais de riscos na gestação $(80,3 \%)$, sobre os riscos de automedicação sem orientação médica durante a gestação $(76,9 \%)$, sobre os malefícios do tabagismo $(75,1 \%)$ e do consumo de álcool durante a gestação $(74,5 \%)$ e a possibilidade de acompanhante no momento do parto $(64,7 \%)$ (Tabela 2). Prevalências abaixo de 50\% foram observadas no recebimento de orientações sobre o manejo adequado da amamentação (45,9\%) e sobre a possibilidade de visitar a maternidade antes do parto (38,2\%). Ao final, observou-se que ter recebido todas as orientações ao menos uma vez durante o pré-natal foi igual a $18,4 \%$ (Tabela 2 ).

Tabela 1. Descrição das características socioeconômicas das participantes do estudo, Santa Catarina, 2019 ( $n=3.111)$.

\begin{tabular}{lcc}
\hline \multicolumn{1}{c}{ Variável } & N & $\%$ \\
\hline Mora marido/companheiro & & \\
\hline Sim & 2529 & 81,3 \\
\hline Não & 582 & 18,7 \\
\hline Idade & & \\
\hline De 14 a 20 anos & 471 & 15,5 \\
\hline De 21 a 24 anos & 619 & 20,4 \\
\hline De 25 a 34 anos & 1480 & 46,2 \\
\hline 35 anos ou mais & 541 & 17,9 \\
\hline Cor da pele & & \\
\hline Branca & 1981 & 63,7 \\
\hline Preta/negra & 283 & 9,1 \\
\hline Parda & 847 & 27,2 \\
\hline Escolaridade & & 10,0 \\
\hline Fundamental incompleto & 565 & 18,2 \\
\hline Fundamental completo & 968 & 31,1 \\
\hline Médio completo & 1267 & 40,7 \\
\hline Superior completo & 311 & \\
\hline Total & & \\
\hline & & \\
\hline
\end{tabular}


Tabela 2. Descrição das características do pré-natal das participantes do estudo, Santa Catarina, 2019 ( $n=3.111)$.

\begin{tabular}{|c|c|c|}
\hline Variável & $\mathbf{N}$ & $\%$ \\
\hline \multicolumn{3}{|c|}{ Profissional que atendeu na maioria do pré-natal } \\
\hline Médico & 1507 & 48,4 \\
\hline Enfermeiro & 355 & 11,4 \\
\hline Enfermeiro e médico & 1249 & 40,2 \\
\hline \multicolumn{3}{|l|}{ № consultas } \\
\hline Até 6 & 611 & 19,6 \\
\hline 7 ou mais & 2500 & 80,4 \\
\hline \multicolumn{3}{|l|}{ Início do pré-natal } \\
\hline$\leq 12$ semanas & 2430 & 78,1 \\
\hline$>12$ semanas & 681 & 21,9 \\
\hline \multicolumn{3}{|c|}{$\begin{array}{l}\text { Orientação sobre amamentação exclusiva } \\
6 \text { meses }\end{array}$} \\
\hline Sim & 1909 & 61,5 \\
\hline Não & 1202 & 38,5 \\
\hline \multicolumn{3}{|c|}{$\begin{array}{l}\text { Orientação quanto ao manejo da } \\
\text { amamentação }\end{array}$} \\
\hline Sim & 1428 & 45,9 \\
\hline Não & 1683 & 54,1 \\
\hline \multicolumn{3}{|c|}{$\begin{array}{l}\text { Orientou sobre importância de atividade física } \\
\text { na gestação }\end{array}$} \\
\hline Sim & 1871 & 60,3 \\
\hline Não & 1240 & 39,7 \\
\hline \multicolumn{3}{|c|}{ Orientou sobre riscos de automedicação } \\
\hline Sim & 2388 & 76,9 \\
\hline Não & 723 & 23,1 \\
\hline \multicolumn{3}{|c|}{ Orientação sobre consumo de álcool } \\
\hline Sim & 2310 & 74,5 \\
\hline Não & 801 & 25,5 \\
\hline \multicolumn{3}{|l|}{ Orientação sobre fumo } \\
\hline Sim & 2331 & 75,1 \\
\hline Não & 780 & 24,9 \\
\hline \multicolumn{3}{|c|}{ Orientaçãos sobre sinais de riscos } \\
\hline Sim & 2489 & 80,3 \\
\hline Não & 622 & 19,7 \\
\hline \multicolumn{3}{|c|}{ Orientação como começa o trabalho de parto } \\
\hline Sim & 1987 & 64,0 \\
\hline Não & 1124 & 36,0 \\
\hline \multicolumn{3}{|c|}{$\begin{array}{l}\text { Orientou sobre a possibilidade de ter } \\
\text { acompanhante no parto }\end{array}$} \\
\hline Sim & 2011 & 64,7 \\
\hline Não & 1100 & 35,3 \\
\hline \multicolumn{3}{|c|}{$\begin{array}{l}\text { Orientou sobre possibilidade de visita à } \\
\text { maternidade }\end{array}$} \\
\hline Sim & 1222 & 39,2 \\
\hline Não & 1889 & 60,8 \\
\hline \multicolumn{3}{|l|}{ Adequação das orientações } \\
\hline Inadequado & 2539 & 81,6 \\
\hline Adequado & 572 & 18,4 \\
\hline Total & 3111 & 100,0 \\
\hline
\end{tabular}

Para as orientações, aquelas atendidas somente por profissional médico receberam menos orientações sobre possibilidade de visita à maternidade $(33,1 \%)$ e mais sobre sinais de riscos $(77,7 \%)$; e aquelas atendidas somente pelo enfermeiro receberam menos orientações quanto ao manejo da amamentação $(42,4 \%)$ e mais sobre sinais de riscos $(80,0 \%)$. Já aquelas atendidas por médico e enfermeiro conjuntamente foram as que menos receberam orientação sobre possibilidade de visita à maternidade $(43,9 \%)$ e mais sobre os sinais de riscos (83,3\%) (Tabela 3).

$\mathrm{Na}$ análise bruta, observou-se que aquelas que tiveram sete ou mais consultas de pré-natal apresentaram 1,29 vez mais chance $(p=0,046)$ de ter adequação de orientação comparadas às que tiveram até seis consultas; e aquelas que iniciaram o pré-natal com mais de 12 semanas apresentaram $10,0 \%$ menos chance de ter adequação nas orientações, se comparadas às que iniciaram antes das 12 semanas, apesar de não haver significância estatística nesta diferença. Ainda, o indicador estado civil demonstrou que aquelas que não residiam com marido ou companheiro apresentaram 1,33 vez mais chance $\left(I_{95 \%} 1,19-1,50\right)$ de ter adequação nas orientações, se comparadas àquelas que residiam.

Em relação à variável desfecho de principal interesse, a análise bruta demostrou que a prevalência de adequação às orientações recebidas no pré-natal foi 1,34 vez maior $(p=0,001)$ entre as mulheres que referiram assistência por médico mais enfermeiro quando comparadas àquelas atendidas somente pelo médico. Não houve diferença entre atendimento majoritário por médico e atendimento majoritário por enfermeiro, apenas (Tabela 4).

Na análise ajustada para adequação de orientações recebidas durante o pré-natal segundo o profissional que atendeu na maioria das consultas, observou-se que aquelas gestantes atendidas na maioria das consultas de pré-natal pelos profissionais médico e também enfermeiro apresentaram chance $41,0 \%$ maior de ter adequação nas orientações, se comparadas àquelas atendidas apenas pelo profissional médico (Tabela 5).

\section{DISCUSSÃO}

Ao analisar os dados do pré-natal no âmbito da Atenção Primária à Saúde no Estado de Santa Catarina, observou-se que a prevalência de adequação das orientações recebidas durante o pré-natal foi de $18,6 \%$, sendo que as orientações recebidas com maior frequência durante o acompanhamento foram relacionadas aos sinais de risco, riscos de automedicação e riscos do fumo. Também se verificou que realizar o acompanhamento conjuntamente com médico e enfermeiro aumentou as chances de adequação às orientações, quando comparado com ter a maior parte das consultas com apenas um profissional.

Em relação às orientações prestadas durante o acompanhamento pré-natal, vale destacar que são práticas profissionais que não implicam custos financeiros adicionais para o SUS, mas dependem de protagonismo e atitudes dos profissionais. Observou-se que, quando analisada a adequação às orientações 
Tabela 3. Descrição das orientações recebidas pelas participantes do estudo, segundo profissional que atendeu na maioria do pré-natal, Santa Catarina, 2019 ( $\mathrm{n=3.111).}$

\begin{tabular}{|c|c|c|c|c|}
\hline Variável & $\begin{array}{l}\text { Médico } \\
\mathrm{n}(\%)\end{array}$ & $\begin{array}{l}\text { Enfermeiro } \\
\mathrm{n}(\%)\end{array}$ & $\begin{array}{l}\text { Médico/Enf } \\
\text { n(\%) }\end{array}$ & p valor* \\
\hline \multicolumn{4}{|l|}{$\begin{array}{l}\text { Orientação sobre amamentação } \\
\text { exclusiva } 6 \text { meses }\end{array}$} & \multirow{3}{*}{0,006} \\
\hline Sim & $749(58,8)$ & $187(60,0)$ & $682(65,2)$ & \\
\hline Não & $527(41,2)$ & $125(40,0)$ & $366(34,8)$ & \\
\hline \multicolumn{4}{|c|}{ Orientação quanto ao manejo da amamentação } & \multirow{3}{*}{0,000} \\
\hline Sim & $526(41,1)$ & $133(42,4)$ & $545(52,0)$ & \\
\hline Não & $750(58,9)$ & $179(57,6)$ & $503(48,0)$ & \\
\hline \multicolumn{4}{|c|}{$\begin{array}{l}\text { Orientou sobre importância de atividade física na } \\
\text { gestação }\end{array}$} & \multirow{3}{*}{0,016} \\
\hline Sim & $743(58,3)$ & $176(56,5)$ & $662(63,4)$ & \\
\hline Não & $533(41,7)$ & $136(43,6)$ & $386(36,6)$ & \\
\hline \multicolumn{4}{|c|}{ Orientou sobre riscos de automedicação } & \multirow{3}{*}{0,155} \\
\hline Sim & $957(75,2)$ & $240(77,1)$ & $821(78,6)$ & \\
\hline Não & $317(24,8)$ & $72(22,9)$ & $227(21,4)$ & \\
\hline \multicolumn{4}{|c|}{ Orientação sobre consumo de álcool } & \multirow{3}{*}{0,003} \\
\hline Sim & $909(71,4)$ & $232(74,3)$ & $812(77,7)$ & \\
\hline Não & $367(28,6)$ & $80(25,7)$ & $236(22,3)$ & \\
\hline \multicolumn{4}{|l|}{ Orientação sobre fumo } & \multirow{3}{*}{0,002} \\
\hline Sim & $918(72,0)$ & $234(75,1)$ & $819(78,4)$ & \\
\hline Não & $358(28,0)$ & $78(24,9)$ & $229(21,6)$ & \\
\hline \multicolumn{4}{|l|}{ Orientação sobre sinais de riscos } & \multirow{3}{*}{0,004} \\
\hline Sim & $989(77,8)$ & $249(80,0)$ & $871(83,3)$ & \\
\hline Não & $287(22,2)$ & $623(20,0)$ & $177(16,7)$ & \\
\hline \multicolumn{4}{|c|}{ Orientação sobre começo do trabalho de parto } & \multirow{3}{*}{0,003} \\
\hline Sim & $781(61,3)$ & $197(63,3)$ & $712(68,1)$ & \\
\hline Não & $495(38,7)$ & $115(36,7)$ & $336(31,9)$ & \\
\hline \multicolumn{4}{|c|}{$\begin{array}{l}\text { Orientou sobre possibilidade de ter } \\
\text { acompanhante no parto }\end{array}$} & \multirow{3}{*}{0,000} \\
\hline Sim & $772(60,6)$ & $192(61,7)$ & $728(69,7)$ & \\
\hline Não & $504(39,4)$ & $120(38,3)$ & $317(30,3)$ & \\
\hline \multicolumn{4}{|c|}{$\begin{array}{l}\text { Orientou sobre possibilidade de visita à } \\
\text { maternidade }\end{array}$} & \multirow{3}{*}{0,000} \\
\hline Sim & $424(33,1)$ & $139(44,6)$ & $560(43,9)$ & \\
\hline Não & $852(66,9)$ & $173(55,4)$ & $588(56,1)$ & \\
\hline \multicolumn{5}{|l|}{ Adequação das orientações } \\
\hline Inadequado & $1072(84,0)$ & $259(83,0)$ & $825(78,7)$ & 0,004 \\
\hline Adequado & $204(16,0)$ & $53(17,0)$ & $223(21,3)$ & \\
\hline
\end{tabular}

*Valor obtido por meio do teste de qui-quadrado. 
Tabela 4. Análise bruta da adequação de orientações recebidas durante o pré-natal, segundo profissional que atendeu na maioria das consultas, Santa Catarina, 2019 ( $n=3.111)$.

\begin{tabular}{|c|c|c|}
\hline \multirow{2}{*}{ Variável } & \multicolumn{2}{|c|}{ Adequado } \\
\hline & OR (IC95\%*) & Valor $p$ \\
\hline \multicolumn{3}{|l|}{ Profissional } \\
\hline Médico & 1,00 & \\
\hline Enfermeiro & $0,96(0,2-1,29)$ & 0,798 \\
\hline Enfermeiro e médico & $1,34(1,19-1,60)$ & 0,001 \\
\hline \multicolumn{3}{|l|}{ Número de consultas } \\
\hline Até 6 & 1,00 & \\
\hline 7 ou mais & $1,29(1,01-1,67)$ & 0,046 \\
\hline \multicolumn{3}{|l|}{ Início pré-natal } \\
\hline$<=12$ semanas & 1,00 & \\
\hline$>12$ semanas & $0,90(0,71-1,15)$ & 0,424 \\
\hline \multicolumn{3}{|l|}{ Mora marido/companheiro } \\
\hline Sim & 1,00 & \\
\hline Não & $1,33(1,08-1,63)$ & 0,006 \\
\hline \multicolumn{3}{|l|}{ Idade } \\
\hline De 14 a 20 anos & 1,00 & \\
\hline De 21 a 24 anos & $0,96(0,70-1,32)$ & 0,846 \\
\hline De 25 a 34 anos & $1,06(0,80-1,39)$ & 0,805 \\
\hline 35 anos ou mais & $0,94(0,67-1,30)$ & 0,723 \\
\hline \multicolumn{3}{|l|}{ Cor pele } \\
\hline Branca & 1,00 & \\
\hline Preta/negra & $1,16(0,86-1,55)$ & 0,315 \\
\hline Parda & $1,01(0,83-1,24)$ & 0,870 \\
\hline \multicolumn{3}{|l|}{ Escolaridade } \\
\hline Fundamental incompleto & 1,00 & \\
\hline Fundamental completo & $0,91(0,69-1,19)$ & 0,471 \\
\hline Médio completo & $0,86(0,66-1,12)$ & 0,282 \\
\hline Superior completo & $1,10(0,76-1,57)$ & 0,633 \\
\hline
\end{tabular}

Tabela 5. Análise ajustada da adequação de orientações recebidas durante o pré-natal, segundo profissional que atendeu na maioria das consultas, Santa Catarina, 2019 ( $n=3.111)$.

\begin{tabular}{|c|c|c|}
\hline \multirow{2}{*}{ Variável } & \multicolumn{2}{|c|}{ Adequado } \\
\hline & OR (IC95\%*) & Valor $\mathrm{p}$ \\
\hline \multicolumn{3}{|l|}{ Profissional } \\
\hline Médico & 1,00 & \\
\hline Enfermeiro & $1,5(0,75-1,47)$ & 0,749 \\
\hline Enfermeiro e Médico & $1,41(1,14-1,75)$ & 0,001 \\
\hline
\end{tabular}

* IC 95\%: intervalo de 95\% de confiança; ** Ajustada por número de consultas, início do pré-natal, mora marido/companheiro, escolaridade, cor da pele e idade. recebidas durante o pré-natal, a maioria apresentou inadequação. Estudo acerca da atenção pré-natal na rede básica de saúde no país ${ }^{7}$ apresentou $39,7 \%$ de inadequação às orientações a partir de dados nacionais do Programa de Melhoria do Acesso e da Qualidade (PMAQ), evidenciando que os serviços ainda não têm conseguido atingir alguns grupos.

O Ministério da Saúde reafirma a importância de os ambientes de saúde estarem abertos para cumprir seu papel de educador e promotor da saúde. Assim, ressalta-se que durante o pré-natal as gestantes e a família devem receber orientações fundamentais para uma adequada atenção ao pré-natal, puerpério e cuidados com o recém-nascido, dentre outras temáticas importantes para a orientação em saúde. ${ }^{12}$

Apesar da relevância das orientações durante o acompanhamento, observou-se neste estudo a baixa prevalência de algumas orientações, sendo que as mais comuns foram sobre os sinais de risco na gestação, riscos de automedicação durante a gestação, riscos do fumo durante a gestação, riscos do consumo de álcool na gestação e sobre a possibilidade de ter acompanhante no parto. Tais achados apontam a existência de um olhar biologicista frente ao pré-natal, no qual ainda é maior a valorização de orientações de riscos em contraposição a orientações de autocuidado e autonomia. Estudo nacional com dados do primeiro ciclo do PMAQ, analisando a prevalência das orientações recebidas no pré-natal, observou que a orientação mais recebida foi acerca da amamentação exclusiva $(91,0 \%)$, seguida da orientação sobre alimentação e ganho de peso $(88,9 \%)$ e cuidados com o bebê $(85,9 \%)$, valores diferentes daqueles encontrados em nosso estudo, o que evidencia lacunas na assistência pré-natal. ${ }^{7}$

O Ministério da Saúde define que, na primeira consulta de pré-natal, devem ser dadas à gestante todas as orientações necessárias para uma gestação saudável, de modo a facilitar sua adesão às condutas e intervenções prescritas, sendo que o sucesso nas orientações é primordial para a adesão da gestante às próximas consultas de pré-natal. ${ }^{12}$ Entretanto, estudo realizado no Rio de Janeiro mostrou que comportamentos de risco, como ingerir bebida alcoólica, fumar e consumir drogas ilícitas durante a gestação foram referidos pelas mulheres em grande quantidade durante a realização do pré-natal em estabelecimentos de saúde públicos, reforçando a importância de tais orientações durante o pré-natal. ${ }^{13}$

Em relação às orientações sobre o manejo da amamentação, observou-se que estas estiveram presentes em apenas $45,9 \%$ dos acompanhamentos, quando deveriam ser reportadas pela totalidade das participantes deste estudo, tendo em vista que a amamentação contribui no fortalecimento de vínculo entre a mãe e o bebê e é muito importante no crescimento e desenvolvimento da criança. Além disso, o período de maior dificuldade para o aleitamento materno ocorre nas primeiras semanas pós-parto, sendo que desconhecimentos a respeito do manejo da amamentação podem ocasionar complicações e levar ao desmame precoce..$^{14}$ 
Para as orientações sobre a possibilidade de visita à maternidade, observou-se que $44,9 \%$ daquelas atendidas por médico e enfermeiro conjuntamente receberam esta orientação, enquanto naquelas atendidas somente pelo médico a prevalência foi de apenas $35,6 \%$. Outro estudo realizado em Vitória-ES, em 2014, demonstrou a importância da orientação e da vinculação da gestante com a maternidade, e que esta deve ocorrer desde o início do acompanhamento pré-natal, facilitando o acesso das gestantes no momento do parto ou em caso de emergências. ${ }^{15}$ Tais orientações surgem a partir da implantação da Rede Cegonha, e têm o propósito de vincular a gestante, evitando peregrinação e dificuldade de acesso, além de agilizar a assistência. ${ }^{16}$

Observou-se maior proporção de ter recebido tais orientações naquelas mulheres que realizaram o pré-natal conjuntamente com os dois profissionais, médico e enfermeiro. Em contrapartida, aquelas atendidas somente pelo profissional médico apresentaram as menores proporções de orientações para visita à maternidade, acompanhante durante o parto, sinais de risco na gestação, risco do fumo, risco do álcool, riscos de automedicação e amamentação exclusiva até os seis meses de idade da criança.

Destaca-se que a prevalência, neste estudo, de sete ou mais consultas no acompanhamento pré-natal foi de $80,9 \%$, em que $78,3 \%$ iniciaram o pré-natal precocemente antes das 12 semanas de gestação. Apesar disso, estudos nacionais, ao avaliar a adequação pré-natal segundo parâmetros do Ministério da Saúde, têm demostrado que apenas 68,3\% apresentaram um pré-natal considerado adequado. ${ }^{17}$ Esses achados apontam um cenário nacional no qual a maioria das gestantes apresentam um número adequado de consultas de pré-natal; no entanto, esse número de consultas não reflete a qualidade destas, e destaca-se a necessidade de avaliar as orientações recebidas durante o pré-natal como critério importante na qualidade da assistência prestada.

O Ministério da Saúde ${ }^{18}$ definiu, dentre as recomendações essenciais no cuidado à saúde da mulher e da criança no âmbito da APS, a importância e a efetividade dos cuidados prestados por mais de um profissional da saúde, como enfermeiros e médicos, na atenção ao pré-natal, perinatal e puerpério, somando-se às contribuições de cada profissão para uma melhor assistência no pré-natal.

Nas gestantes atendidas apenas pelo profissional enfermeiro, observou-se maior proporção de orientações sobre os sinais de risco na gestação, riscos do fumo, álcool e riscos de automedicação. De acordo com a Lei do Exercício Profissional da Enfermagem (Lei no 7.498/1986) e o Ministério da Saúde, o pré-natal de baixo risco pode ser acompanhado pelo enfermeiro no âmbito da APS, cabendo ao profissional realizar atividades de educação em saúde à gestante e à puérpera, prestando, a partir da consulta de enfermagem, a prescrição e a assistência de enfermagem. ${ }^{19}$ Como reflexo disso, a formação em Enfermagem permite atuar na assistência ao pré-natal, realizando ações educativas individuais e em grupo e na assistência de enfermagem como membro da equipe. ${ }^{20}$

Nas análises, observou-se que a adequação às orientações se manteve associada com o profissional, sendo maior naquelas atendidas conjuntamente pelo profissional médico e enfermeiro. Observa-se, portanto, que cada profissional possui suas especificidades no atendimento às gestantes e, por meio da união de profissionais com conhecimentos distintos e que se complementam, os benefícios são observados na assistência a esse público, contribuindo também na melhoria dos indicadores de saúde da mulher e da criança.

Assim, conforme as competências dispostas no Caderno de Atenção ao Pré-natal de Baixo Risco, do Ministério da Saúde, cabe aos profissionais médicos e enfermeiros participarem como membros da equipe na assistência ao pré-natal, contribuindo para a promoção da saúde da gestante e a melhoria na sua qualidade de vida, exercendo, assim, papel educativo. ${ }^{12}$

Tais achados levam a uma reflexão acerca do processo de trabalho na Atenção Primária, em que se destacam as ações de saúde de forma integrada, com continuidade do atendimento. Desta forma, reafirma-se a importância do profissional médico e do enfermeiro enquanto membros da equipe na assistência à mulher e ao bebê de acordo com suas especificidades, na busca da melhoria da assistência prestada e no protagonismo da mulher.

A presente investigação tem limitações que merecem ser destacadas. Uma delas refere-se ao viés de memória frente às informações referentes ao pré-natal. As puérperas podem ter apresentado dificuldade em responder a algumas perguntas relativas ao pré-natal. No entanto, o período recordatório não é muito extenso, e a gravidez tende a ser momento marcante, que se reflete em boa recordação dos eventos. Além disso, aplicou-se questionário testado para auxiliar na obtenção de respostas adequadas, e os entrevistadores foram extensivamente treinados para a melhor aplicação do mesmo. Há de se destacar, também, a alta taxa de resposta obtida e a elevada amostra analisada

\section{CONCLUSÕES E IMPLICAÇÕES PARA A PRÁTICA}

Os resultados deste estudo demonstraram a existência de lacunas em relação às orientações que são ofertadas pelos profissionais de saúde durante o acompanhamento pré-natal. Em contrapartida, observou-se que a adequação às orientações recebidas durante o pré-natal é maior quando há atenção compartilhada entre médicos e enfermeiros.

Ao analisar as orientações, observou-se baixa prevalência de oferta de algumas recomendações, perdendo-se oportunidades importantes para sensibilizar e empoderar as gestantes quanto ao acesso a informações que podem contribuir para uma boa gestação, parto e puerpério. Devem-se definir métodos e estratégias, a fim de garantir que a gestante receba todas as orientações preconizadas e tenha possibilidade de executá-las. 
Assim, reforça-se a importância dos atendimentos compartilhados durante o pré-natal e na promoção de ações educativas para o esclarecimento de todas as suas dúvidas e inseguranças, contribuindo para uma gestação de baixo risco, tanto para a mulher quanto para a criança.

A partir dos objetivos deste estudo, foi possível conhecer a atuação compartilhada dos profissionais enfermeiros e médicos frente às orientações prestadas durante o pré-natal. Identificou-se que a atuação compartilhada por meio de um processo sistematizado de assistência pode promover melhores desfechos no acompanhamento ao pré-natal, parto e puerpério. Entretanto, é necessário dar continuidade aos estudos sobre a saúde da mulher e da criança, buscando-se estratégias de intervenções e orientações para a garantia de uma atenção mais equânime e resolutiva.

\section{FINANCIAMENTO}

Fundação de Amparo à Pesquisa de Santa Catarina (FAPESC). Termo de outorga $n^{\circ} 2017$ TR1364; projeto de pesquisa "Pré-Natal e puerpério imediato na Atenção Básica: avaliação da gestão da Rede Cegonha em Santa Catarina", coordenado por Antonio Fernando Boing.

\section{CONTRIBUIÇÕES DOS AUTORES}

Desenho do estudo. Coleta, análise de dados e interpretação dos resultados. Redação e revisão crítica do manuscrito. Aprovação da versão final do artigo. Responsabilidade por todos os aspectos do conteúdo e a integridade do artigo publicado. Bruna Leticia Marques. Yaná Tamara Tomasi. Suelen dos Santos Saraiva.

Desenho do estudo. Coleta de dados. Redação e revisão crítica do manuscrito. Aprovação da versão final do artigo. Responsabilidade por todos os aspectos do conteúdo e a integridade do artigo publicado. Antonio Fernando Boing.

Análise de dados e interpretação dos resultados. Redação e revisão crítica do manuscrito. Aprovação da versão final do artigo. Responsabilidade por todos os aspectos do conteúdo e a integridade do artigo publicado. Daniela Savi Geremia.

\section{EDITOR ASSOCIADO}

Gerson Luiz Marinho

\section{REFERÊNCIAS}

1. Instituto Brasileiro de Geografia e Estatística. Tábua completa de mortalidade para o Brasil - 2018. Rio de Janeiro: IBGE; 2019.

2. United Nations. The Millennium Development Goals Report 2015. New York: United Nations; 2015.

3. Barros FC, Bhutta ZA, Batra M, Hansen TN, Victora CG, Rubens CE. Global report on preterm birth and stillbirth (3 of 7): evidence for effectiveness of interventions. BMC Pregnancy Childbirth. 2010 fev;10(Suppl 1):S3. http://dx.doi.org/10.1186/1471-2393-10-S1-S3. PMid:20233384.
4. Bhutta ZA, Das JK, Bahl R, Lawn JE, Salam RA, Paul VK et al. Can available interventions end preventable deaths in mothers, newborn babies, and stillbirths, and at what cost? Lancet. 2014 jul;384(9940):34770. http://dx.doi.org/10.1016/S0140-6736(14)60792-3. PMid:24853604.

5. Domingues RMSM, Viellas EF, Dias MAB, Torres JA, Theme-Filha MM, Gama SGN et al. Adequação da assistência pré-natal segundo as características maternas no Brasil. Rev Panam Salud Publica. 2015;37(3):140-7. PMid:25988250.

6. Carroli G, Rooney C, Villar J. How effective is antenatal care in preventing maternal mortality and serious morbidity? An overview of the evidence. Paediatr Perinat Epidemiol. 2001;15(Suppl 1):1-42. http://dx.doi. org/10.1046/j.1365-3016.2001.00001.x. PMid:11243499.

7. Tomasi E, Fernandes PAA, Fischer T, Siqueira FCV, Silveira DSD, Thumé $E$ et al. Qualidade da atenção pré-natal na rede básica de saúde do Brasil: indicadores e desigualdades sociais. Cad Saude Publica. 2017;33(3):e00195815. http://dx.doi.org/10.1590/0102-311x00195815. PMid:28380149.

8. Carvalho DSC, Novaes HMD. Avaliação da implantação de programa de atenção pré-natal no Município de Curitiba, Paraná, Brasil: estudo em coorte de primigestas. Cad Saude Publica. 2004;20(Suppl 2):S220-30. http://dx.doi.org/10.1590/S0102-311X2004000800017.PMid:15608936.

9. Matos DS, Rodrigues MS, Rodrigues TS. Atuação do enfermeiro na assistência ao pré-natal de baixo risco na estratégia saúde da família em um município de Minas Gerais. Rev Enfermagem. [Internet] 2013 [citado 1 mar 2020];16(1):18-33. Disponível em: http://periodicos. pucminas.br/index.php/enfermagemrevista/article/ view/5282/5237

10. Silva SR, Parreira BDM, Dias FA, Cardoso LE, Cunha JD. Práticas de autocuidado desenvolvidas por gestantes atendidas em um ambulatório de pré-natal. Rev Eletr Enf. 2014;16(4):812-21. http://dx.doi.org/10.5216/ ree.v16i4.21779.

11. Reserch Eletronic Data Capture. First Latin American and Brazilian REDCapCon [Internet]. [citado 1 mar 2020]. Disponível em: http://www. redcapbrasil.com.br/redcapcon/en/redcapcon/en/

12. Ministério da Saúde (BR). Atenção ao pré-natal de baixo. Brasília: Ministério da Saúde; 2012.

13. Leal MDC, Gama SGND, Campos MR, Cavalini LT, Garbayo LS, Brasi $\mathrm{CLP}$ et al. Fatores associados à morbi-mortalidade perinatal em uma amostra de maternidades públicas e privadas do Município do Rio de Janeiro, 1999-2001. Cad Saude Publica. 2004;20(Suppl 1):S20-33. http://dx.doi.org/10.1590/S0102-311X2004000700003.

14. Ministério da Saúde (BR). Saúde da criança: aleitamento materno e alimentação complementar. 2a ed. Brasília: Ministério da Saúde;2015.

15. Polgliane RBS, Leal MDC, Amorim MHC, Zandonade E, Santos Neto ETD. Adequação do processo de assistência pré-natal segundo critérios do Programa de Humanização do Pré-natal e Nascimento e da Organização Mundial de Saúde. Cien Saude Colet. 2014;19(7):19992010. http://dx.doi.org/10.1590/1413-81232014197.08622013.

16. Portaria $\mathrm{n}=1.459$, de 24 de junho de 2011 (BR). Institui, no âmbito do Sistema Único de Saúde (SUS) a Rede Cegonha. Diário Oficial da União [periódico na internet], Brasília (DF), 24 jun 2011 [citado 1 mar 2020]. Disponível em: https://bvsms.saude.gov.br/bvs/saudelegis/ gm/2011/prt1459_24_06_2011.html

17. Viellas EF, Domingues RMSM, Dias MAB, Gama SGN, Theme MM Fa, Costa JV et al. Assistência pré-natal no Brasil. Cad Saude Publica. 2014;30(Suppl 1):S85-100. http://dx.doi.org/10.1590/0102-311X00126013.

18. Ministério da Saúde (BR). O SUS de A a Z: garantindo saúde nos municípios. 3a ed. Brasília: Ministério da Saúde; 2009.

19. Santos EF, Santos EB, Santana GO, Assis MFD, Meneses RDO. Legislação em enfermagem: atos normativos do exercício e do ensino de enfermagem. Rio de Janeiro: Atheneu; 1997.

20. Rodrigues EM, Nascimento RG, Araújo A. Protocolo na assistência prénatal: ações, facilidades e dificuldades dos enfermeiros da Estratégia de Saúde da Família. Rev Esc Enferm USP. 2011;45(5):1041-7. http:// dx.doi.org/10.1590/S0080-62342011000500002. PMid:22031361.

aExtraído do trabalho de conclusão de curso "Assistência pré-natal na Atenção Primária: Um olhar sob a atuação do profissional enfermeiro no estado de Santa Catarina". Autoria de: Bruna Letícia Marques. Orientação da Professora Yaná Tamara Tomasi. Curso de Graduação em Enfermagem. Universidade Federal da Fronteira Sul (UFFS), 2019. 